\title{
Utilização de Medidas Biométricas para Predizer Características da Carcaça de Cabritos Saanen ${ }^{1}$
}

\author{
Enrique Alejandro Yáñez ${ }^{2,4}$, Kleber Tomás de Resende ${ }^{3}$, Ângela Cristina Dias Ferreira ${ }^{4}$, \\ Ariosvaldo Nunes Medeiros ${ }^{5}$, Américo Garcia da Silva Sobrinho ${ }^{3}$, José Morais Pereira Filho ${ }^{6}$, \\ Izabelle Auxiliadora Molina de Almeida Teixeira ${ }^{7}$, Silvana Martinez Baraldi Artoni ${ }^{8}$
}

RESUMO - Foram determinadas medidas biométricas de caprinos leiteiros com o objetivo de estimar equações que permitam predizer o peso e as características da carcaça de animais sob diferentes condições nutricionais. Realizou-se um experimento em duas fases, utilizando-se 27 cabritos machos da raça Saanen em cada uma, distribuídos nos tratamentos: alimentação à vontade, 30 e $60 \%$ de restrição. O peso vivo (PV) inicial foi de $5 \mathrm{~kg}$ na Fase 1 e $20 \mathrm{~kg}$ na Fase 2, abatendo-se os animais ao atingirem 20 e $35 \mathrm{~kg}$ de PV, respectivamente. Foram determinadas as medidas biométricas e avaliada a condição corporal no animal vivo e, após o abate e resfriamento de 24 horas, as medidas da carcaça. Estimaram-se o peso em jejum (PJ), o peso da carcaça fria (PCF) e a compacidade da carcaça, em função das medidas biométricas. O perímetro torácico e o comprimento corporal foram as medidas biométricas que apresentaram melhor ajuste para estimar o PJ, o PCF e a compacidade da carcaça e, por isso, são recomendados, por serem precisos, práticos e de fácil execução.

Palavras-chave: biometria, caprinos, compacidade, comprimento corporal, condição corporal, perímetro torácico

\section{Utilization of Biometric Measures for Prediction of Saanen Goats Carcass Traits}

\begin{abstract}
Biometric measures of dairy goats were taken to estimate equations for prediction of weight and carcass characteristics of animals with different nutritional conditions. An experiment was carried out into two phases, with 27 male Saanen goats at each one. The animals were assigned to the following feeding groups: ad libitum, 30 and $60 \%$ of restriction. The initial live weight (LW) was $5 \mathrm{~kg}$ at the Phase 1 and $20 \mathrm{~kg}$ at the Phase 2, the animals were slaughtered when they reached 20 and $35 \mathrm{~kg}$ of LW, respectively. The biometric measurements and body conditions were determined when the animals were alive. After the slaughter and 24 hours of chilling, were evaluated the carcass characteristics. The fasted live weight (FLW), cold carcass weight $(\mathrm{CCW})$ and carcass compactness were estimated in function of the biometric measurements. Among the biometric measurements, thoracic perimeter and body length showed the best adjustment for FLW, CCW and compactness estimation, and are recommended, because of its precision and easy execution.
\end{abstract}

Key Words: biometric, body length, compactness, goats, score corporal, thoracic circumference

\section{Introdução}

A criação de caprinos no Brasil é desenvolvida principalmente em sistema extensivo - a maioria de pequenos produtores que, normalmente, contam com pouca tecnologia e baixo investimento em infraestrutura, inviabilizando o controle de desempenho a partir da pesagem periódica dos animais. Destaca-se, também, a necessidade de contar com mensurações no animal vivo que permitam predizer características quantitativas da carcaça, rendimento, conformação e proporção de cortes.

A metodologia utilizada deve ter como premissa a confiabilidade das medidas a serem usadas em equações de predição para estimar parâmetros no animal vivo ou na carcaça, que diferem com a raça, idade, sexo e estado nutricional dos caprinos. É razoável pensar que medidas isoladas não sejam

\footnotetext{
${ }^{1}$ Parte da tese de Doutorado em Zootecnia-Produção Animal do primeiro autor. Projeto financiado junto à FAPESP pelo segundo autor. 2 Professor do Departamento de Producción Animal da Facultad de Ciencias Veterinarias, UNNE, Corrientes. Sargento Cabral 2139 , Corrientes, Argentina. CP: 3400 (eyanez@vet.unne.edu.ar).

${ }_{3}^{3}$ Professor do Departamento de Zootecnia da Faculdade de Ciências Agrárias e Veterinárias da Unesp, Jaboticabal. Rod. P.D. Castellane s/n, Jaboticabal, SP, Brasil. CEP: 14884-900 (kresende@fcav.unesp.br). Bolsista do CNPq.

4 Professora da UNOESTE, SP (acrisdias@yahoo.com.br)

5 Professor do Departamento de Zootecnia do Centro de Ciências Agrárias da UFPB, Areia, PB, Brasil.

6 Professor do Departamento de Zootecnia UFPB, Patos, PB (jmorais@cstr.ufcg.edu.br).

7 Pesquisadora vinculada ao Departamento de Zootecnia da Faculdade de Ciências Agrárias da Unesp, Jaboticabal, SP (izabelle@fcav.unesp.br). 8 Professora do Departamento de Morfologia e Fisiologia da FCAV, Unesp, Jaboticabal, SP.
} 
suficientes para definir as características da carcaça, mas combinações permitirão estabelecer índices para comparação das carcaças.

Resende et al. (2001), trabalhando com cabras Saanen, utilizaram a biometria para estimar o peso vivo (PV), correlacionando este com o perímetro torácico, comprimento e altura do animal. Os trabalhos de Valdez et al. (1982), Mohamed \& Amin (1996) e Varade et al. (1997) mostraram alta correlação entre PV e medidas biométricas.

Outro parâmetro que deve ser avaliado na venda dos caprinos é o rendimento comercial de carcaça, pois sua comercialização se realiza na expectativa do produto que poderá chegar ao consumidor. Além disso, características da carcaça como compacidade, perímetro da garupa e da perna, entre outros, poderão influenciar o preço de venda do produto, modificando a percepção visual do consumidor e favorecendo o consumo de carne caprina.

As informações da literatura sugerem não haver padronização entre as metodologias utilizadas pelos diferentes autores, sendo poucos os trabalhos desenvolvidos visando correlacionar medidas biométricas com características da carcaça de caprinos leiteiros. Nesse sentido, objetivou-se, com este trabalho, desenvolver, a partir de mensurações simples e econômicas no animal vivo, equações de predição do peso e de características da carcaça de cabritos leiteiros submetidos a diferentes condições nutricionais.

\section{Material e Métodos}

O experimento foi realizado no Setor de Caprinocultura da Faculdade de Ciências Agrárias e Veterinárias-Unesp, Jaboticabal, SP, Brasil, sendo desenvolvido em duas fases:

Fase 1: utilizaram-se 27 cabritos inteiros da raça Saanen, com peso vivo (PV) inicial de $5 \mathrm{~kg}$, divididos em nove grupos de três animais, alocados completamente ao acaso em três tratamentos: $\mathrm{T}_{1}: 0 \%$ de restrição (alimentação à vontade) $\mathrm{T}_{2}: 30 \%$ de restrição e $\mathrm{T}_{3}: 60 \%$ de restrição. Quando os animais alimentados à vontade atingiram $20 \mathrm{~kg}$ de $\mathrm{PV}$, todo o grupo correspondente era abatido;

Fase 2: utilizaram-se 27 animais castrados da raça Saanen, com PV inicial de $20 \mathrm{~kg}$, distribuídos nos mesmos tratamentos da Fase 1. Quando os animais alimentados à vontade atingiram $35 \mathrm{~kg}$ de $\mathrm{PV}$, todo o grupo correspondente era abatido.
Os animais receberam a mesma dieta nas duas Fases, a qual foi constituída por $46,9 \%$ de feno de planta de milho e $53,1 \%$ de concentrado formado por $25,9 \%$ de milho moído, $19,3 \%$ de farelo de soja, $4,3 \%$ de melaço de cana de açúcar, 2,0\% de núcleo mineral, $0,8 \%$ de óleo de soja e $0,8 \%$ de calcário calcítico; obtendo uma ração com 16,5\% de proteína bruta, 2,46 Mcal de energia metabolizável e $48,8 \%$ de fibra em detergente neutro, por $\mathrm{kg}$ de matéria seca. A composição bromatológica dos alimentos apresenta-se na Tabela 1.

Na Fase 1, foi oferecido aos cabritos leite de vaca, permitindo ingestões máximas de 1,50, 1,05 e $0,60 \mathrm{~L} /$ dia para 0,30 e $60 \%$ de restrição, respectivamente, sendo desaleitados aos 50 dias de idade. A dieta sólida foi oferecida a partir dos 7 dias de vida, sendo pesadas diariamente as sobras para ajuste das quantidades fornecidas para cada nível alimentar.

Todos os animais da Fase 2 receberam até 1,5 L de leite de vaca/dia, sendo desaleitados aos 50 dias, e dieta sólida à vontade até os $20 \mathrm{~kg}$ de PV. Os animais que compuseram esta Fase foram castrados com $17 \mathrm{~kg}$ de PV, em média, em virtude da expectativa de que atingiriam o peso de abate após os 4 meses, idade em que as características organolépticas da carne podem ser alteradas pelo início da puberdade.

Ao atingir o peso de abate, os animais foram submetidos a jejum de alimentos sólidos por 24 horas e de água por 16 horas, caracterizando o peso vivo em jejum (PJ). Antes do abate foram determinadas, com os animais em pé sobre superfície plana, as medidas biométricas: Comprimento corporal (CC): distância entre a articulação cérvico-torácica e a base da cauda na primeira articulação intercoccígea; Comprimento da perna: distância entre o trocânter maior do fêmur e o bordo da articulação tarsometatarsiana; Altura do anterior: distância entre a região da cernelha e a extremidade distal do membro anterior; Altura do posterior: distância entre a tuberosidade sacra e a extremidade distal do membro posterior; Perímetro torácico: perímetro tomando-se como base o esterno e a cernelha, passando a fita métrica detrás da paleta; Perímetro da perna: perímetro tomando como base a parte média da perna, acima da articulação fêmuro-tibio-rotuliana; Largura da garupa: distância entre os trocânteres maiores dos fêmures; Largura do peito: distância entre as faces laterais das articulações escápuloumerais.

\section{R. Bras. Zootec., v.33, n.6, p.1564-1572, 2004}


Foi estimada também a compacidade corporal (COMPAC), índice objetivo da conformação in vivo, obtido pela fórmula: $\mathrm{COMPAC}=\mathrm{PJ} / \mathrm{CC}(\mathrm{kg} / \mathrm{cm})$, sendo que quanto maior a compacidade corporal, maior a proporção de músculos e gordura no animal. Foi avaliada a condição corporal dos animais por apalpação da região do lombo e do esterno, utilizando-se como resultado final a média das duas notas, seguindo a metodologia descrita por Morand-Fehr \& Herviev (1989).

O abate foi realizado por insensibilização com descarga elétrica, seguida de sangria. Após a esfola e evisceração, obteve-se o peso da carcaça quente (PCQ), a qual foi colocada em câmara fria $\left(4^{\circ} \mathrm{C}\right)$ por $24 \mathrm{~h}$ para se obter o peso de carcaça fria (PCF).

Nas carcaças, foram realizadas as seguintes mensurações: Comprimento externo da carcaça (CEC): distância entre a articulação cérvico-torácica e a $1^{\mathrm{a}}$ articulação intercoccígea; Comprimento interno da carcaça (CIC): distância entre o bordo anterior do osso púbis e o bordo anterior da primeira costela em seu ponto médio; Comprimento da perna: distância entre o trocânter maior do fêmur e o bordo da articulação tarso-metatarsiana; Largura da garupa: largura máxima entre os trocânteres dos fêmures; Perímetro da garupa: perímetro na região da garupa, com base nos trocânteres dos fêmures; Perímetro da perna: perímetro tomando como base a parte média da perna, acima da articulação fêmuro-tibio-rotuliana; Perímetro do tórax: perímetro medido detrás da paleta; Profundidade do tórax: distância entre o esterno e a cernelha; Largura do tórax: largura máxima do tórax. Também foram determinados a compacidade da carcaça: $\mathrm{COC}=\mathrm{PCF} / \mathrm{CIC},(\mathrm{kg} / \mathrm{cm})$, e o rendimento comercial $=$ PCF/PJ $(\%)$.

O delineamento para cada fase foi inteiramente casualizado, com três tratamentos e nove repetições. As médias foram comparadas pelo teste Tukey a 5\%. Foram estimadas equações de regressão para predição das características da carcaça, considerando a totalidade dos animais das duas fases.

\section{Resultados e Discussão}

Na Fase 1, os animais alimentados à vontade alcançaram $21 \mathrm{~kg}$ em 76 dias de experimento, com 90 dias de idade, em média, ao passo que, na Fase 2, os animais alimentados à vontade atingiram $35,2 \mathrm{~kg}$ de PV em 68 dias de experimento, com 167 dias de idade, em média. Na Tabela 2, constam ganho de peso diário, PV, PJ, PCF, rendimento e condição corporal.

As diferenças no peso e no score de condição corporal dos animais demonstram que a restrição

Tabela 1 - Composição percentual dos alimentos experimentais (\% MS)

Table 1 - Percentage composition of the experimental feedstuffs (\% DM)

\begin{tabular}{|c|c|c|c|c|c|c|}
\hline $\begin{array}{l}\text { Ingrediente } \\
\text { Ingredient }\end{array}$ & $\begin{array}{l}\text { MS } \\
D M\end{array}$ & $\begin{array}{l}\mathrm{EM}^{1} \\
M E^{1}\end{array}$ & $\begin{array}{l}\mathrm{PB} \\
C P\end{array}$ & $\begin{array}{l}\mathrm{EE} \\
E E\end{array}$ & $\begin{array}{l}\mathrm{FDN} \\
N D F\end{array}$ & $\begin{array}{l}\text { FDA } \\
A D F \\
\end{array}$ \\
\hline $\begin{array}{l}\text { Feno da planta de milho } \\
\text { Maiz hay }\end{array}$ & 91,21 & 2,27 & 6,49 & 0,78 & 84,63 & 17,34 \\
\hline $\begin{array}{l}\text { Milho moído } \\
\text { Ground corn }\end{array}$ & 90,30 & 3,22 & 10,50 & 3,50 & 20,61 & 1,57 \\
\hline $\begin{array}{l}\text { Farelo de soja } \\
\text { Soybean meal }\end{array}$ & 90,57 & 3,18 & 54,58 & 0,50 & 12,84 & 9,67 \\
\hline $\begin{array}{l}\text { Melaço } \\
\text { Molasse }\end{array}$ & 87,56 & 2,60 & 3,46 & - & - & - \\
\hline $\begin{array}{l}\text { Óleo de soja } \\
\text { Soybean oil }\end{array}$ & 99,00 & 9,00 & - & - & - & - \\
\hline $\begin{array}{l}\text { Núcleo mineral }^{2} \\
\text { Mineral mixture }^{2}\end{array}$ & 94,20 & - & - & - & - & - \\
\hline $\begin{array}{l}\text { Calcário calcítico } \\
\text { Limestone }\end{array}$ & 99,99 & - & - & - & - & - \\
\hline
\end{tabular}

$1 \mathrm{Mcal} / \mathrm{kg}$ de MS (Mcal/kg DM).

2 Núcleo mineral (quantidade/kg do produto): Cálcio 190 g; Fósforo 73 g; Sódio 62 g; Cloro 90 g; Magnésio $44 \mathrm{~g}$; Enxofre $30 \mathrm{~g}$; Zinco 1350 mg; Cobre 340 mg; Manganês 940 mg; Ferro 1064 mg; Cobalto $3 \mathrm{mg}$; lodo $16 \mathrm{mg}$; Selênio $18 \mathrm{mg}$; Flúor máx. $730 \mathrm{mg}$.

2 Mineral salt (amount/kg of product): Calcium $190 \mathrm{~g}$; Phosphorus $73 \mathrm{~g}$; Sodium $62 \mathrm{~g}$; Chlorine $90 \mathrm{~g}$; Magnesium $44 \mathrm{~g}$; Sulfur $30 \mathrm{~g}$; Zinc 1350 mg; Copper 340 mg; Manganese 940 mg; Iron 1064 mg; Cobalt 3 mg; lodine 16 mg; Selenium $18 \mathrm{mg}$; Fluoride máx. $730 \mathrm{mg}$. 
alimentar afetou o desenvolvimento e a deposição de tecidos, obtendo no final do período experimental cabritos com diferentes pesos e condição nutricional.

O baixo rendimento comercial da carcaça dos cabritos alimentados à vontade e abatidos com $21 \mathrm{~kg}$ de PV reflete a especialização destes na produção de leite e o fato de se encontrarem na fase inicial de desenvolvimento, com predomínio de crescimento dos tecidos ósseo e muscular e pouca deposição de gordura, além da maior participação do trato gastrintestinal (TGI), como demonstrado por Yáñez (2002). O maior rendimento observado na Fase 2, pode ser atribuído à idade e ao PV, devendo-se considerar também que os animais desta Fase eram castrados e apresentaram maior proporção de gordura na carcaça (Yáñez, 2002), o que influi diretamente no rendimento da mesma.

As medidas biométricas são apresentadas na Tabela 3. A largura da garupa apresentou o mesmo comportamento, em ambas as fases, com valores semelhantes para os tratamentos com 0 e $30 \%$ de restrição, sendo ambos superiores a $60 \%$ de restrição $(\mathrm{P}<0,05)$. Esta semelhança entre os animais alimentados à vontade e com $30 \%$ de restrição pode ser atribuída à base óssea da medida, associada ao pequeno desenvolvimento muscular da região, característico de animais com biótipo funcional leiteiro. Percebe-se, ainda, que o aumento na largura da garupa foi muito pequeno após os $21 \mathrm{~kg}$ de PV.

Na Fase 2, observou-se que as médias dos tratamentos à vontade e $30 \%$ de restrição foram semelhantes para a maioria das variáveis $(\mathrm{P}>0,05)$, com diferenças entre os três tratamentos apenas para perímetro do tórax e compacidade corporal $(\mathrm{P}<0,05)$. Estes resultados podem ser explicados pelas mesmas condições de crescimento dos cabritos até os $20 \mathrm{~kg}$ de PV, e como as medidas utilizadas têm principalmente base óssea, a restrição de $30 \%$ não prejudicou o crescimento deste tecido, de desenvolvimento precoce.

No caso do perímetro torácico, medida influenciada pela base óssea e muscular, bem como por depósitos de gordura, a deposição de tecido adiposo, principalmente na região do esterno, deve ter determinado as diferenças entre tratamentos.

A compacidade corporal é um índice que estima objetivamente a conformação nos animais vivos, a partir de dois valores fáceis de determinar com precisão, o comprimento corporal e o PJ. A compacidade corporal foi diferente entre todos os

Tabela 2 - Médias e desvios-padrão do peso vivo (PV), ganho de peso diário (GPD), peso em jejum (PJ), peso da carcaça fria (PCF), rendimento comercial de carcaça (PCF/PJ) e condição corporal, de cabritos submetidos a três níveis de restrição alimentar

Table 2 - Means and standard deviations of live weight (LW), daily weight gain (WG), fast weight (FW), cold carcass weight (CCW), commercial dressing out (CCW/FW) and score corporal of kids submitted to three alimentary restrictions levels

\begin{tabular}{|c|c|c|c|c|c|c|}
\hline $\begin{array}{l}\text { Tratamento } \\
\text { Treatments }\end{array}$ & $\begin{array}{c}\mathrm{PV}(\mathrm{kg}) \\
L W\end{array}$ & $\begin{array}{c}\mathrm{GPD}(\mathrm{g} / \mathrm{dia}) \\
W G \\
(g / d a y)\end{array}$ & $\begin{array}{l}\text { PJ } \\
F W \\
(k g)\end{array}$ & $\begin{array}{l}\mathrm{PCF} \\
C C W \\
(k g)\end{array}$ & $\begin{array}{l}\text { Rendimento } \\
\text { Dressing out } \\
\quad(\%)\end{array}$ & $\begin{array}{c}\text { Condição corporal } \\
\text { Body score }\end{array}$ \\
\hline FASE 1 & & & & & & \\
\hline $\begin{array}{l}\boldsymbol{P H A S E} \mathbf{1} \\
0 \% \text { restrição } \\
0 \% \text { restriction }\end{array}$ & $21,0 \pm 0,36 \mathrm{a}$ & $213,3 \pm 26,5 \mathrm{a}$ & $19,8 \pm 0,51 \mathrm{a}$ & $8,7 \pm 0,54 \mathrm{a}$ & $43,7 \pm 2,41 \mathrm{a}$ & $2,35 \pm 0,24 \mathrm{a}$ \\
\hline $\begin{array}{l}30 \% \text { restriçãão } \\
30 \% \text { restriction }\end{array}$ & $16,2 \pm 1,25 b$ & $148,4 \pm 19,5 b$ & $15,6 \pm 1,17 b$ & $6,6 \pm 0,71 b$ & $42,4 \pm 2,07 \mathrm{ab}$ & $1,96 \pm 0,31 b$ \\
\hline $\begin{array}{l}60 \% \text { restrição } \\
60 \% \text { restriction } \\
\end{array}$ & $11,1 \pm 0,83 c$ & $79,9 \pm 11,1 \mathrm{c}$ & $10,6 \pm 0,96 \mathrm{c}$ & $4,3 \pm 0,34 \mathrm{c}$ & $40,9 \pm 1,72 b$ & $1,44 \pm 0,25 \mathrm{c}$ \\
\hline FASE 2 & & & & & & \\
\hline $\begin{array}{l}\boldsymbol{P H A S E} 2 \\
0 \% \text { restrição } \\
\text { 0\% restriction }\end{array}$ & $35,2 \pm 0,26 a$ & $217,8 \pm 25,1 \mathrm{a}$ & $32,9 \pm 0,54 \mathrm{a}$ & $15,5 \pm 0,58 \mathrm{a}$ & $47,3 \pm 2,07 \mathrm{a}$ & $2,49 \pm 0,36 \mathrm{a}$ \\
\hline $\begin{array}{l}30 \% \text { restrição } \\
30 \% \text { restriction }\end{array}$ & $28,6 \pm 1,31 b$ & $120,2 \pm 17,2 b$ & $27,7 \pm 1,46 b$ & $13,1 \pm 0,89 b$ & $47,2 \pm 2,76 \mathrm{a}$ & $2,01 \pm 0,27 b$ \\
\hline $\begin{array}{l}60 \% \text { restrição } \\
60 \% \text { restriction }\end{array}$ & $22,1 \pm 0,65 c$ & $27,6 \pm 8,60 \mathrm{c}$ & $21,1 \pm 0,57 \mathrm{c}$ & $9,3 \pm 0,57 \mathrm{c}$ & $43,8 \pm 2,27 b$ & $1,26 \pm 0,27 \mathrm{c}$ \\
\hline
\end{tabular}

Médias na mesma coluna em cada fase, seguidas por letras diferentes, diferem $(P<0,05)$ pelo teste Tukey.

Means in the same column in each phase, followed by different letters, differ $(P<.05)$ by Tukey test.

R. Bras. Zootec., v.33, n.6, p.1564-1572, 2004 
tratamentos nas duas fases, coincidindo com o observado para desempenho e condição corporal dos animais.

Além das medidas biométricas descritas foi determinado o perímetro da perna. Esta medida não foi obtida com exatidão, em razão dos movimentos e dos diferentes estados de contração muscular dos animais, fatos que modificam sensivelmente o valor $\mathrm{e}$ que resultaram em dados pouco coerentes e bastante variáveis. Infere-se, portanto, que esta medida não é confiável para estimar a musculosidade desta região, sendo desconsiderada.
Na carcaça (Tabela 4), o perímetro torácico e a largura do tórax diferiram nos tratamentos em ambas as fases $(\mathrm{P}<0,05)$. Todavia, a profundidade do tórax não apresentou diferenças entre os tratamentos com 0 e $30 \%$ de restrição, fato difícil de explicar, pois esperava-se comportamento semelhante às outras medidas torácicas.

A largura de garupa na carcaça não diferiu entre 0 e $30 \%$ de restrição, confirmando o observado no animal vivo. Todavia o perímetro da garupa foi estatisticamente diferente entre os tratamentos, nas duas fases, demonstrando que, ao utilizar uma medida

Tabela 3 - Médias e desvios-padrão das medidas biométricas $(\mathrm{cm})$, de cabritos submetidos a três níveis de restrição alimentar

Table 3 - Means and standard deviations of biometrics measures (cm), of submitted kids to three feeding restrictions levels

\begin{tabular}{lcccc}
\hline $\begin{array}{l}\text { Tratamentos } \\
\text { Treatments }\end{array}$ & $\begin{array}{c}\text { Comprimento corporal } \\
\text { Bodylength }\end{array}$ & $\begin{array}{c}\text { Comprimento perna } \\
\text { Leg length }\end{array}$ & $\begin{array}{c}\text { Altura anterior } \\
\text { Anterior height }\end{array}$ & $\begin{array}{c}\text { Altura posterior } \\
\text { Hind height }\end{array}$ \\
$\begin{array}{l}\text { FASE 1 } \\
\text { PHASE 1 }\end{array}$ & & & \\
$\begin{array}{l}0 \% \text { restrição } \\
0 \% \text { restriction }\end{array}$ & $55,8 \pm 1,6 \mathrm{a}$ & $36,7 \pm 1,6 \mathrm{a}$ & $59,9 \pm 1,6 \mathrm{a}$ & $56,9 \pm 1,7 \mathrm{a}$ \\
$\begin{array}{l}30 \% \text { restrição } \\
30 \% \text { restriction }\end{array}$ & $50,7 \pm 1,5 \mathrm{~b}$ & $34,8 \pm 1,5 \mathrm{~b}$ & $57,3 \pm 2,3 \mathrm{~b}$ & $54,1 \pm 2,3 \mathrm{~b}$ \\
$60 \%$ restrição & $46,0 \pm 2,0 \mathrm{c}$ & $31,1 \pm 0,7 \mathrm{c}$ & $52,0 \pm 1,5 \mathrm{c}$ & $49,2 \pm 2,1 \mathrm{c}$
\end{tabular}

$60 \%$ restriction

\section{FASE 2 \\ PHASE 2}

$0 \%$ restrição

$0 \%$ restriction

$30 \%$ restrição

$30 \%$ restriction

$60 \%$ restrição

$60 \%$ restriction

$\begin{array}{llll}64,2 \pm 2,7 \mathrm{a} & 34,7 \pm 1,3 \mathrm{a} & 68,3 \pm 1,8 \mathrm{a} & 64,9 \pm 1,2 \mathrm{a} \\ 61,9 \pm 1,0 \mathrm{a} & 33,9 \pm 1,5 \mathrm{a} & 66,6 \pm 2,0 \mathrm{ab} & 64,0 \pm 1,6 \mathrm{a} \\ 58,8 \pm 2,0 \mathrm{~b} & 31,8 \pm 1,8 \mathrm{~b} & 64,4 \pm 2,1 \mathrm{~b} & 61,4 \pm 1,8 \mathrm{~b}\end{array}$

\begin{tabular}{|c|c|c|c|c|}
\hline & $\begin{array}{l}\text { Largura peito } \\
\text { Breast width }\end{array}$ & $\begin{array}{l}\text { Largura garupa } \\
\text { Hind width }\end{array}$ & $\begin{array}{c}\text { Perímetro tórax } \\
\text { Thoracic circumference }\end{array}$ & $\begin{array}{c}\text { Compacidade corporal* } \\
\text { Body compactness }\end{array}$ \\
\hline \multicolumn{5}{|l|}{ FASE 1} \\
\hline $\begin{array}{l}\text { PHASE } 1 \\
0 \% \text { restrição } \\
\text { 0\% restriction }\end{array}$ & $16,6 \pm 1,2 \mathrm{a}$ & $14,0 \pm 0,7 \mathrm{a}$ & $61,5 \pm 1,4 \mathrm{a}$ & $0,36 \pm 0,01 \mathrm{a}$ \\
\hline $\begin{array}{l}30 \% \text { restrição } \\
30 \% \text { restriction }\end{array}$ & $14,9 \pm 1,4 b$ & $13,2 \pm 0,7 \mathrm{a}$ & $57,8 \pm 1,4 b$ & $0,31 \pm 0,02 b$ \\
\hline $\begin{array}{l}60 \% \text { restrição } \\
60 \% \text { restriction }\end{array}$ & $12,4 \pm 0,5 \mathrm{c}$ & $11,8 \pm 1,1 \mathrm{~b}$ & $51,6 \pm 2,1 \mathrm{~b}$ & $0,23 \pm 0,01 \mathrm{c}$ \\
\hline
\end{tabular}

\section{FASE 2}

PHASE 2

$0 \%$ restrição

$0 \%$ restriction

$30 \%$ restrição

$30 \%$ restriction

$60 \%$ restrição

$60 \%$ restriction
$19,8 \pm 1,0 \mathrm{a}$

$18,1 \pm 1,7 \mathrm{~b}$
$16,9 \pm 1,0 \mathrm{~b}$
$15,9 \pm 0,5 \mathrm{a}$

$15,4 \pm 1,6 \mathrm{ab}$

$14,3 \pm 0,5 b$
$72,7 \pm 1,8 \mathrm{a}$

$0,51 \pm 0,02 \mathrm{a}$

$68,2 \pm 1,7 b$

$0,45 \pm 0,03 \mathrm{~b}$

Compacidade corporal: peso em jejum/comprimento corporal $(\mathrm{kg} / \mathrm{cm})$.

Médias na mesma coluna em cada fase, seguidas por letras diferentes, diferem $(P<0,05)$ pelo teste Tukey.

${ }^{*}$ Body compactness: fast weight/body length $(\mathrm{kg} / \mathrm{cm})$.

Means in the same column in the phase followed by different letters differ $(P<.05)$ by Tukey test. 
de base principalmente muscular, as diferenças no status nutricional são evidentes e confirmam os resultados verificados na condição corporal, na compacidade corporal e na compacidade da carcaça. Estas observações permitem inferir que a largura de garupa não é bom indicador das diferenças de estado nutricional em animais com pouca massa muscular nesta região. Porém, poderá ser útil em animais de corte, nos quais a musculatura é mais desenvolvida e será influenciada pela condição nutricional.

As outras medidas na carcaça mantiveram comportamento semelhante às medidas biométricas. A compacidade refletiu as diferenças observadas para o peso da carcaça e o comprimento interno, mostrando sua correlação com a conformação da carcaça. Além disso, essas diferenças entre tratamentos para compacidade da carcaça indicam que a restrição alimentar provocou menor deposição de tecidos muscular e adiposo, como observado pela condição corporal e compacidade corporal (Tabelas 2 e 3), confirmando a importância da estimativa da compacidade da carcaça na predição da qualidade da mesma.

A partir das medidas observadas no animal vivo e na carcaça, foram estimadas equações de regressão para predizer o PJ, PCF, rendimento comercial e

Tabela 4 - Médias e desvios-padrão das medidas da carcaça (cm) de cabritos submetidos a três níveis de restrição alimentar

Table 4 - Means and standard deviations of carcass measurements $(\mathrm{cm})$ of kids submitted to three alimentary restrictions levels Tratamento Comprimento externo Comprimento interno Comprimento perna Perímetro perna Largura garupa Treatments External length Internal length Leglength $\quad$ Leg circumference Hindwidth

\begin{tabular}{|c|c|c|c|c|c|}
\hline \\
\hline \multicolumn{6}{|l|}{ PHASE 1} \\
\hline $\begin{array}{l}0 \% \text { restrição } \\
0 \% \text { restriction }\end{array}$ & $50,0 \pm 1,9 \mathrm{a}$ & $57,2 \pm 2,2 \mathrm{a}$ & $34,4 \pm 0,9 \mathrm{a}$ & $24,0 \pm 1,6 \mathrm{a}$ & $13,9 \pm 2,3 \mathrm{a}$ \\
\hline $\begin{array}{l}30 \% \text { restrição } \\
30 \% \text { restriction }\end{array}$ & $46,1 \pm 2,3 b$ & $51,6 \pm 1,8 b$ & $33,1 \pm 1,8 \mathrm{a}$ & $21,7 \pm 1,1 b$ & $12,5 \pm 1,7 \mathrm{a}$ \\
\hline $\begin{array}{l}60 \% \text { restrição } \\
60 \% \text { restriction }\end{array}$ & $41,6 \pm 1,2 \mathrm{c}$ & $46,0 \pm 1,5 \mathrm{c}$ & $30,4 \pm 1,7 \mathrm{~b}$ & $18,7 \pm 1,3 \mathrm{c}$ & $10,4 \pm 0,8 b$ \\
\hline \multicolumn{6}{|l|}{ FASE 2} \\
\hline $\begin{array}{l}0 \% \text { restrição } \\
0 \% \text { restriction }\end{array}$ & $60,3 \pm 1,6 a$ & $64,5 \pm 1,5 \mathrm{a}$ & $38,6 \pm 0,8 \mathrm{a}$ & $30,3 \pm 1,6 \mathrm{a}$ & $14,0 \pm 0,6 \mathrm{a}$ \\
\hline $\begin{array}{l}30 \% \text { restrição } \\
30 \% \text { restriction }\end{array}$ & $58,1 \pm 1,9 \mathrm{ab}$ & $61,0 \pm 1,3 b$ & $36,4 \pm 3,5 \mathrm{ab}$ & $28,7 \pm 1,7 \mathrm{a}$ & $13,5 \pm 0,7 \mathrm{a}$ \\
\hline $60 \%$ restrição & $55,7 \pm 2,9 b$ & $59,2 \pm 1,9 b$ & $35,8 \pm 1,0 \mathrm{~b}$ & $25,1 \pm 0,6 \mathrm{~b}$ & $12,6 \pm 0,5 b$ \\
\hline
\end{tabular}

$60 \%$ restriction

\begin{tabular}{|c|c|c|c|c|}
\hline & $\begin{array}{l}\text { Perímetro tórax } \\
\text { Thoracic perimeter }\end{array}$ & $\begin{array}{l}\text { Largura tórax } \\
\text { Thoracic width }\end{array}$ & $\begin{array}{l}\text { Profundidade tórax } \\
\text { Thoracic depth }\end{array}$ & $\begin{array}{c}\text { Compacidade * } \\
\text { Compactness }\end{array}$ \\
\hline \multicolumn{5}{|l|}{ FASE 1} \\
\hline PHASE 1 & & & & \\
\hline $\begin{array}{l}0 \% \text { restrição } \\
0 \% \text { restriction }\end{array}$ & $58,5 \pm 1,0 \mathrm{a}$ & $16,7 \pm 1,6 \mathrm{a}$ & $21,8 \pm 0,9 \mathrm{a}$ & $0.15 \pm 0,010 \mathrm{a}$ \\
\hline $\begin{array}{l}30 \% \text { restrição } \\
30 \% \text { restriction }\end{array}$ & $54,8 \pm 2,2 b$ & $14,1 \pm 0,9 b$ & $21,7 \pm 0,5 \mathrm{a}$ & $0.13 \pm 0,014 b$ \\
\hline $\begin{array}{l}60 \% \text { restrição } \\
60 \% \text { restriction } \\
\end{array}$ & $49,3 \pm 1,6 \mathrm{c}$ & $12,1 \pm 1,1 \mathrm{c}$ & $20,5 \pm 0,7 b$ & $0.10 \pm 0,005 \mathrm{c}$ \\
\hline \multicolumn{5}{|l|}{$\overline{\text { FASE } 2}$} \\
\hline PHASE 2 & & & & \\
\hline $\begin{array}{l}0 \% \text { restrição } \\
0 \% \text { restriction }\end{array}$ & $68,8 \pm 1,8 \mathrm{a}$ & $22,4 \pm 1,2 \mathrm{a}$ & $26,0 \pm 1,4 \mathrm{a}$ & $0,24 \pm 0,014 \mathrm{a}$ \\
\hline $\begin{array}{l}30 \% \text { restrição } \\
30 \% \text { restriction }\end{array}$ & $64,9 \pm 1,0 \mathrm{~b}$ & $19,7 \pm 2,1 b$ & $25,1 \pm 1,3 \mathrm{ab}$ & $0,21 \pm 0,015 b$ \\
\hline $\begin{array}{l}60 \% \text { restrição } \\
60 \% \text { restriction }\end{array}$ & $60,4 \pm 2,2 \mathrm{c}$ & $17,5 \pm 1,3 \mathrm{c}$ & $24,0 \pm 1,5 \mathrm{~b}$ & $0,16 \pm 0,012 \mathrm{c}$ \\
\hline
\end{tabular}

* Compacidade da carcaça: peso da carcaça fria/comprimento interno da carcaça $(\mathrm{kg} / \mathrm{cm})$.

Médias na mesma coluna em cada fase, seguidas por letras diferentes, diferem $(P<0,05)$ pelo teste Tukey.

${ }^{*}$ Carcass compactness: cold carcass weight/carcass internal length $(\mathrm{kg} / \mathrm{cm})$.

Means in the same column in the phase, followed by different letters differ $(P<.05)$ by Tukey test.

R. Bras. Zootec., v.33, n.6, p.1564-1572, 2004 
outras características da carcaça, em função das medidas biométricas, utilizando os dados dos animais dos três tratamentos e de ambas as fases. Entre as equações estatisticamente significativas, foram selecionadas aquelas que apresentaram maior precisão para estimar os dados observados (coeficiente de determinação) e com explicação e aplicação biológica (Tabela 5). Algumas equações estatisticamente significativas e com alto $\mathrm{R}^{2}$ foram excluídas por sua falta de correlação biológica entre a medida na carcaça e a medida biométrica em função da qual foi estimada. Cita-se, como exemplo, a estimativa do comprimento interno da carcaça, em função da altura.

Tabela 5 - Equações de regressão para estimar o peso em jejum (PJ) e da carcaça fria (PCF), comprimento interno (CIC), compacidade da carcaça (COC), perímetro da garupa (PGAR) e da perna (PPER) na carcaça, em função de medidas biométricas e do PJ, de cabritos submetidos a três níveis de restrição alimentar, incluindo os animais das duas fases do experimento

Table 5 - Regressions equations for estimating fast weight (FW), cold carcass weight (CCW), carcass internal length (CIL), carcass compactness (CC), hind circumference (HC) and leg circumference (LC) in carcass, in function of biometric measures and of FW, of submitted kids to three alimentary restrictions levels, included animals for two experimental phases

\begin{tabular}{|c|c|c|c|}
\hline $\begin{array}{l}\text { Equação } \\
\text { Equations }\end{array}$ & $\begin{array}{l}\text { Probabilidade } \\
\text { Probability }\end{array}$ & $\begin{array}{l}\mathrm{R}^{2} \\
(\%)\end{array}$ & $\begin{array}{l}\mathrm{CV} \\
(\%)\end{array}$ \\
\hline $\mathrm{PJ}=-38,2836+1,0613 \mathrm{CC}$ & 0,0001 & 88,24 & 12,15 \\
\hline $\begin{array}{l}F W=-38.2836+1.0613 B L \\
\mathrm{PJ}=-0,2672-0,3677 \mathrm{PT}+0,0113 \mathrm{PT}^{2} \\
F W=-.2672-.3677 \mathrm{TC}+.0113 \mathrm{TC}^{2}\end{array}$ & 0,0100 & 95,44 & 7,64 \\
\hline $\begin{array}{l}\mathrm{PJ}=-49,5655+1,1535 \mathrm{AA} \\
F W=-49.5655+1.1535 \mathrm{AH}\end{array}$ & 0,0001 & 84,63 & 13,89 \\
\hline $\begin{array}{l}\mathrm{PCF}=-20,6059+0,5377 \mathrm{CC} \\
C C W=-20.6059+.5377 B L\end{array}$ & 0,0001 & 85,70 & 15,32 \\
\hline $\begin{array}{l}\mathrm{PCF}=9,1608-0,5347 \mathrm{PT}+0,0085 \mathrm{PT}^{2} \\
C C W=9.1608-.5347 T C+0,0085 T C^{2}\end{array}$ & 0,0002 & 95,54 & 8,63 \\
\hline $\begin{array}{l}\mathrm{PCF}=-5,2354+40,0739 \text { COMPAC } \\
C C W=-5.2354+40.0739 \text { BOCOM }\end{array}$ & 0,0001 & 95,90 & 8,20 \\
\hline $\begin{array}{l}\mathrm{PCF}=-1,2632+0,5091 \mathrm{PJ} \\
C C W=-1.2632+.5091 \mathrm{FW}\end{array}$ & 0,0001 & 98,07 & 5,63 \\
\hline $\begin{array}{l}C I C=-43,2034+2,6858 C C-0,0160 C^{2} \\
C I L=-43.2034+2.6858 B L-.0160 B L^{2}\end{array}$ & 0,0133 & 91,38 & 3,39 \\
\hline $\begin{array}{l}\mathrm{CIC}=11,90134+184,8391 \text { COMPAC }-162,8410 \mathrm{COMPAC}^{2} \\
C I L=11.90134+184.8391 \text { BOCOM }-162.8410 \text { BOCOM}^{2}\end{array}$ & 0,0001 & 89,47 & 3,75 \\
\hline $\begin{array}{l}\mathrm{CIC}=27,0177+2,0911 \mathrm{PJ}-0,0295 \mathrm{PJ}^{2} \\
C I L=27.0177+2.0911 F W-0,0295 F W^{2}\end{array}$ & 0,0001 & 93,18 & 3,01 \\
\hline $\begin{array}{l}\mathrm{COC}=-0,2277+0,0070 \mathrm{CC} \\
C C=-.2277+.0070 \mathrm{BL}\end{array}$ & 0,0001 & 81,76 & 13,39 \\
\hline $\begin{array}{l}\mathrm{COC}=0,1774-0,0077 \mathrm{PT}+0,00012 \mathrm{PT}^{2} \\
C C=.1774-.0077 T C+0,00012 T C^{2}\end{array}$ & 0,0001 & 94,04 & 7,73 \\
\hline $\begin{array}{l}\mathrm{COC}=-0,0305+0,5274 \text { COMPAC } \\
C C=-.0305+.5274 \text { BOCOM } \\
\mathrm{COC}=0,0225+0,0067 \mathrm{PJ}\end{array}$ & 0,0001 & 93,86 & 7,77 \\
\hline$C C=.0225+.0067 F W$ & 0,0001 & 95,07 & 6,96 \\
\hline $\begin{array}{l}\text { PGAR }=12,9078+124,0566 \text { COMPAC }-67,8817 \text { COMPAC }^{2} \\
H C=12.9078+124.0566 \text { BOCOM }-67.8817 \text { BOCOM }^{2}\end{array}$ & 0,0001 & 93,81 & 3,69 \\
\hline $\begin{array}{l}\mathrm{PGAR}=29,0851+0,9307 \mathrm{PJ} \\
H C=29.0851+.9307 \mathrm{FW}\end{array}$ & 0,0001 & 95,59 & 3,08 \\
\hline $\begin{array}{l}\mathrm{PPER}=9,5640+41,0623 \mathrm{COMPAC} \\
L C=9.5640+41.0623 \text { BOCOM }\end{array}$ & 0,0001 & 84,59 & 6,71 \\
\hline
\end{tabular}

PJ e PCF em kg; Compacidade da carcaça: PCF / CIC $(\mathrm{kg} / \mathrm{cm})$

CC: comprimento corporal; AA: altura do anterior; PT: perímetro torácico; COMPAC: compacidade corporal (PJ / CC, em kg/cm)

FW and CCW in kg; Carcass compactness: CCW / CIL

BL: Body length, AH: anterior height, TC: thoracic circumference; BOCOM: body compactness (FW/BL, in $\mathrm{kg} / \mathrm{cm}$ )

R. Bras. Zootec., v.33, n.6, p.1564-1572, 2004 
O peso em jejum é a forma mais confiável de avaliar o peso do animal vivo, por diminuir as variações decorrentes das diferenças no conteúdo do trato digestório, o qual é influenciado pelo tipo de alimento e sistema de alimentação. As equações para predizer o rendimento comercial em função das medidas biométricas apresentaram menor $\mathrm{R}^{2}$ (abaixo de 60\%) que aquelas que estimaram o PJ e o PCF, sendo desconsideradas.

As medidas perímetro da garupa e da perna na carcaça podem ser utilizadas como indicativo da conformação e da musculosidade da perna, apesar da ausência de pesquisas correlacionando estas medidas com a composição tecidual e/ou participação proporcional desta região na carcaça de caprinos.

O perímetro torácico apresentou-se como a melhor opção para predizer o PJ, o PCF e a compacidade da carcaça (Tabela 5), por apresentar equações com altos $\mathrm{R}^{2}$, baixos $\mathrm{CV}$ e serem significativas $(\mathrm{P}<0,01)$. Os resultados das equações em função do comprimento corporal apresentaram menor $\mathrm{R}^{2} \mathrm{e}$ maior CV. A compacidade corporal e o PJ apresentaram bom ajuste para determinar o PCF, o comprimento interno, a compacidade e o perímetro da garupa na carcaça. O PJ poderá ser utilizado a partir de seu valor real ou, ainda, estimado em função do PT ou CC.

Os resultados obtidos neste experimento corroboram os apresentados por Resende et al. (2001), que reportaram que o perímetro torácico foi a medida biométrica de maior correlação com o PV, ao trabalharem com fêmeas Saanen em aleitamento, recria, gestação, lactação e secas. A equação em função do PT foi a que melhor se ajustou à curva de crescimento desses animais. Os autores concluíram que o PT pode ser utilizado para predizer o PV a partir de uma equação geral para todas as idades.

Estes resultados também são semelhantes aos obtidos por Valdez et al. (1982), trabalhando com caprinos de diferentes grupos raciais e sexo e com ampla variação de peso e idade, que observaram que o PT foi a melhor medida para estimar o PV. Da mesma forma, Mohamed \& Amin (1996) recomendaram a utilização do perímetro torácico para determinar o PV de caprinos de diferentes pesos e categorias zootécnicas, destacando que a equação em função do PT apresentou melhor ajuste ao ser aplicada para caprinos jovens.

Os resultados obtidos em função do PT, neste experimento, apresentaram melhor ajuste que os observados com equação similar por Varade et al.
(1997), que verificaram $R^{2}$ de $73 \%$, trabalhando com fêmeas caprinas, com PV médio de 26,4 kg e 1,5 ano de idade. Os autores concluíram que a equação para estimar o PV em função do perímetro torácico apresentou melhor ajuste que as obtidas com comprimento corporal e perímetro abdominal, e consideraram prática sua utilização nas condições de produção. Estes resultados diferem daqueles de Osório et al. (1999), ao trabalharem com cordeiros Corriedale abatidos com 9,5 meses de idade, que observaram ser o peso vivo a variável de maior relação com as medidas na carcaça. O perímetro torácico foi, na ordem, a quarta variável melhor correlacionada.

Considerando que a combinação das medidas poderia oferecer melhor ajuste dos dados, estimou-se equações de regressão múltipla para predizer o PJ, o PCF e a compacidade da carcaça, em função de medidas biométricas. Foram selecionadas estas variáveis, por serem as mais importantes na avaliação da produção (PJ, PCF), e a compacidade, por predizer com precisão a quantidade de músculo da carcaça (Yáñez, 2002).

As equações de regressão múltipla apresentaram pequeno aumento no $\mathrm{R}^{2}$, em relação ao ajuste verificado para as equações obtidas em função do perímetro torácico e do peso em jejum, demonstrando que a realização de mais uma mensuração traz poucos benefícios, não justificados pelo aumento de trabalho e tempo requeridos. Valdez et al. (1982) e Resende et al. (2001) afirmaram que a utilização de equações de regressão em função de várias medidas biométricas produzem pequeno aumento na precisão das estimativas obtidas, o que não justificaria, na prática, o uso de mais medidas além do PT.

A possibilidade de predizer o peso da carcaça fria permite estimar o rendimento comercial, indicador de características quantitativas da carcaça. O rendimento é influenciado pela deposição de gordura, conformação e musculosidade da carcaça, além da idade e dos estados fisiológico e nutricional do animal. Yáñez (2002) observou que a restrição alimentar reduziu o peso da carcaça fria, o rendimento comercial e a proporção de gordura da carcaça, diminuindo também a proporção de músculos nos cabritos mais jovens, podendo-se inferir que, quanto maior o rendimento, maior a proporção de músculos e de gordura da carcaça.

Com a utilização de mensurações de fácil execução e baixo custo, e a partir das equações apresentadas, o produtor ou comprador de caprinos poderá estimar

\section{R. Bras. Zootec., v.33, n.6, p.1564-1572, 2004}


o peso do animal, os kg de produto disponíveis para a comercialização e a compacidade da carcaça, medida objetiva da conformação que poderá influenciar o preço de venda e a procura do consumidor. Destaca-se também a importância da aplicação destas mensurações no controle zootécnico do rebanho, permitindo ao produtor com menos recursos a avaliação da produtividade de sua propriedade.

\section{Conclusões}

As equações estimadas em função do perímetro torácico e do comprimento corporal predizem com precisão o peso em jejum, o peso da carcaça fria e a compacidade da carcaça de cabritos leiteiros, com 11 a $35 \mathrm{~kg}$ de peso vivo e com diferentes condições corporais.

A compacidade corporal estima adequadamente o peso da carcaça fria e a compacidade da carcaça destes animais.

\section{Literatura Citada}

MOHAMED, I.D.; AMIN, J.D. Estimating body weight from morphometric measurements of Sahell (Borno Withe) goats. Small Ruminant Research, v.24, p.1-5, 1996.

MORAND-FEHR, P.; HERVIEV, J. Noctatión de l'état corpore: a vos stylos!. La Chevre, n.175, p.39-42, 1989.
OSÓRIO, J.C.; OSÓRIO, M.T.; VAZ, C.M. et al. Coeficientes de correlación entre medidas "in vivo" y de la canal en ovinos de la raza criolla. In: Congreso Latinoamericano de Especialistas en Pequeños Rumiantes y Camélidos Sudamericanos, 1., 1999, Montevideo, Uruguay. Memórias.... Montevideo: Sociedad Latinoamericana de Especialistas en Pequeños Rumiantes y Camélidos Sudamericanos, 1999. CD-ROM.

RESENDE, K.T.; MEDEIROS, A.N.; CALEGARI, A. et al. Utilización de medidas corporales para estimar el peso vivo de caprinos Saanen. In: JORNADAS CIENTÍFICAS, 26.; INTERNACIONALES DE LA SOCIEDAD ESPAÑOLA DE OVINOTECNIA Y CAPRINOTECNIA, 5., 2001, Sevilla, España. Memorias... Sevilla: Sociedad Española de Ovinotecnia y Caprinotecnia, 2001. p.340-344.

VALDEZ, C.A.; FAGAN,D.V.; VICERA, I.B. The correlation of body weight to esternal body measurements in goats. Philippine Journal of Animal Industry, v.37, n.4, p.62-89, 1982.

VARADE, P.K.; ALI, S.Z.; MALKHEDE, P.S. Body measurements of local goats under field conditions. Indian Veterinary Journal, v.74, p.448-449, 1997.

YÁÑEZ, E.A. Desenvolvimento tecidual e características da carcaça de cabritos Saanen, com diferentes pesos e níveis nutricionais. Jaboticabal: Universidade Estadual Paulista, 2002. 85p. Tese (Doutorado em Zootecnia) Universidade Estadual Paulista, 2002.

Recebido em: 17/02/03

Aceito em: 19/02/04 